\title{
The Influence of Acoustic Impedance Mismatch on Post-Stenotic Pulsed-Doppler Ultrasound Measurements in a Coronary Artery
}

\section{Model}

\section{S. A. Jones}

John Hopkins University

Follow this and additional works at: https://engagedscholarship.csuohio.edu/encbe_facpub

Leclere Georgia Institute of Technology

How does access to this work benefit you? Let us know!

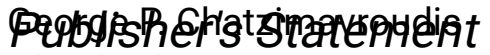

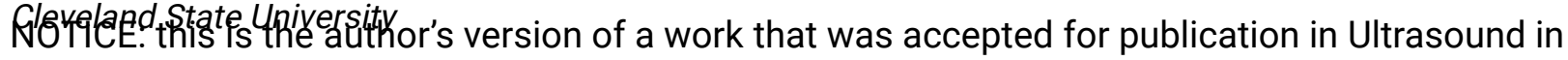

Meplikin and Biology. Changes resulting from the publishing process, such as peer review,

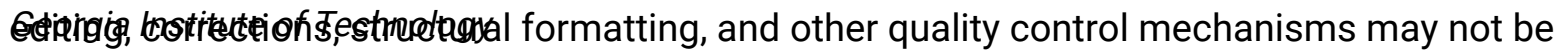
reflected in this document. Changes may have been made to this work since it was submitted EAr Biology, 22, 5, (1996)] DOI 10.1016/0301-5629(96)00025-7

See next page for additional authors Original Citation

Jones SA, Leclerc H, Chatzimavroudis GP, Y. HK, Scott NA, Yoganathan AP. The influence of acoustic impedance mismatch on post-stenotic pulsed-Doppler ultrasound measurements in a coronary artery model. Ultrasound Med Biol. 1996;22:623-634.

Repository Citation

Jones, S. A.; Leclerc, H.; Chatzimavroudis, George P.; Kim, Y. H.; Scott, N. A.; and Yoganathan, Ajit P., "The Influence of Acoustic Impedance Mismatch on Post-Stenotic Pulsed-Doppler Ultrasound Measurements in a Coronary Artery Model" (1996). Chemical \& Biomedical Engineering Faculty Publications. 98.

https://engagedscholarship.csuohio.edu/encbe_facpub/98

This Article is brought to you for free and open access by the Chemical \& Biomedical Engineering Department at EngagedScholarship@CSU. It has been accepted for inclusion in Chemical \& Biomedical Engineering Faculty Publications by an authorized administrator of EngagedScholarship@CSU. For more information, please contact library.es@csuohio.edu. 


\section{Authors}

S. A. Jones, H. Leclerc, George P. Chatzimavroudis, Y. H. Kim, N. A. Scott, and Ajit P. Yoganathan

This article is available at EngagedScholarship@CSU: https://engagedscholarship.csuohio.edu/encbe_facpub/98 


\title{
THE INFLUENCE OF ACOUSTIC IMPEDANCE MISMATCH ON POST- STENOTIC PULSED-DOPPLER ULTRASOUND MEASUREMENTS IN A CORONARY ARTERY MODEL
}

\author{
S. A. Jones, ${ }^{\dagger}$ H. LeClerc, ${ }^{\ddagger}$ G. P. Chatzimavroudis, ${ }^{*}$ Y. H. KIM, ${ }^{*}$ \\ N. A. SCOTT ${ }^{\S}$ and A. P. Yoganathan* \\ ${ }^{\dagger}$ Department of Biomedical Engineering, Johns Hopkins University School of Medicine, Baltimore, MD, USA; \\ ${ }^{\ddagger}$ George Woodruff School of Mechanical Engineering, *Cardiovascular Fluid Mechanics Laboratory, \\ School of Chemical Engineering, Georgia Institute of Technology, Atlanta, GA, USA; \\ and ${ }^{8}$ Interventional Cardiology, Emory University Hospital, Atlanta, GA, USA
}

\begin{abstract}
Acoustic impedance mismatch at the fluid-wall interface was shown to affect the spectra from an intravascular Doppler device in an in vitro model with a diameter typical of human coronary arteries. Measurements were obtained first under Poiseuille flow conditions with impedance mismatches of $0 \%, 7 \%$ and $12 \%$, and then under stenosed conditions for the $0 \%$ and $7 \%$ mismatch cases. For the zero mismatch case, the Doppler spectra could be readily interpreted in terms of fluid mechanical phenomena. When mismatch was present, the spectra from Poiseuille flow exhibited multiple peaks which could not be directly related to the velocity profile. Also, the spectra from stenosed flow with a mismatch of $7 \%$ were similar to those from the zero mismatch case but did not exhibit the specific flow-related features as clearly. These results indicate that the impedance mismatch alters the acoustic environment inside the model and that this causes artifact in the Doppler spectra.
\end{abstract}

Key Words: Acoustics, Artery, Beam pattern, Blood, Coronary, Diagnosis, Doppler, Guidewire, Impedance, Spectrum, Stenosis, Ultrasound, Velocity.

\section{INTRODUCTION}

The consequences of severe coronary artery stenosis include high pressure drops across the lesion and consequent reductions in coronary blood flow reserve. Sites of stenosis are also susceptible to plaque rupture and subsequent thrombosis (Chesebro et al. 1991). The diagnosis and treatment of these lesions is thus a fundamental problem in interventional cardiology. Treatments include open heart surgery and percutaneous transluminal coronary angioplasty. The former is highly invasive, which means that a clear diagnosis of lesion severity is needed before it is undertaken. The latter, though less invasive, involves a $5 \%$ probability of acute closure (Stewart 1991), mainly as a result of thrombosis, and a $40 \%$ probability (Eisenberg 1993) of restenosis. Thus, there is a need to determine the factors which contribute to these complications.
Although the biochemical pathways that lead to thrombosis are well studied, it is not known what triggers these pathways at the stenosis site. One hypothesis is that the flow patterns within and downstream of the stenosis are partially responsible, since it is known that regions of stagnant flow can lead to thrombosis (Dintenfass 1964). The mechanisms by which restenosis occurs are similarly unknown, but since regions of low shear stress and recirculation have been associated with intimal hyperplasia (Bassiouny et al. 1992) and atherosclerosis ( $\mathrm{Ku}$ et al. 1985), a hemodynamic mechanism can again be postulated. Angiography, the method most often used in the catheterization laboratory to diagnose stenoses, yields good estimates of the lesion diameter, but does not provide direct information about the flow patterns. It is reasonable to believe that this flow information can be obtained through Doppler ultrasound methods. Specifically, it should be contained in the power spectra of the quadrature signals from an appropriate Doppler catheter or guidewire. The most important part of the spectrum in this 
case is the lower frequency range, which corresponds to low velocities, and hence stagnation, recirculation, and low hemodynamic wall shear stress.

Currently several types of intracoronary Doppler instruments are commercially available. Most of these are designed to have a high carrier frequency, typically $20 \mathrm{MHz}$, and a small sample volume. However, the Cardiometrics guidewire (Mountain View, CA) has a relatively smaller crystal $(<0.5 \mathrm{~mm}$ diameter $)$ and lower carrier frequencies $(12-14 \mathrm{MHz})$. These parameters result in a wider beam pattern which provides a more uniform beam over the cross-section of the artery, and this is important if different regions of the spectra are to be compared.

The wider beam pattern, however, increases the potential for distortion of the beam by the interface between the artery wall and the blood, where the acoustic impedance can change. It is thus important to design in vitro models to accurately account for this. Also, since the mismatch in acoustic impedance between blood and the arterial wall can range from $3.7 \%-6.9 \%$ in normal humans (Greenleaf et al. 1974), it is important to determine to what extent this mismatch can affect the Doppler ultrasound signals in vivo.

The experiments discussed here address some of these questions. First, the existence of mismatch phenomena is studied in steady Poiseuille flow; the power spectra are examined for mismatches of $12 \%, 7 \%$ and $0 \%$. Next, for zero mismatch, power spectra are ob-

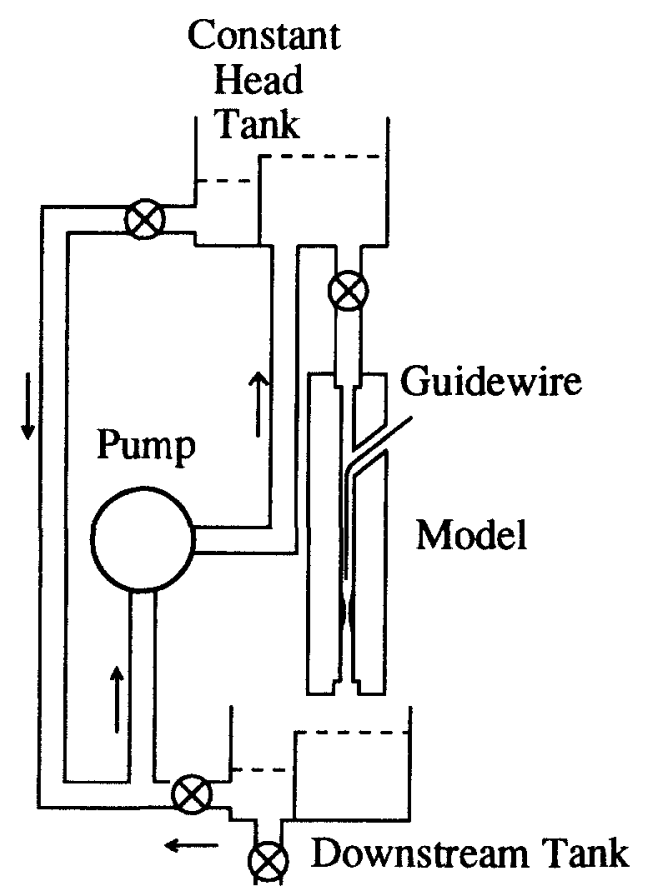

Fig. 1. Experimental setup for the Doppler ultrasound measurements. tained from three stenosis models with cross-sectional area reductions of $50 \%, 75 \%$ and $90 \%$ under Reynolds numbers, which correspond to mean and peak coronary flow rates. These experiments are then repeated with $7 \%$ mismatch for the $50 \%$ and $75 \%$ area reductions.

\section{METHODS}

The model used in this work is a straight 3.18$\mathrm{mm}$ diameter cylindrical tube cast within a $5.2 \times 5.1$ $\mathrm{cm}$ rigid block. It was $35-\mathrm{cm}$ long to ensure fully developed flow at the entrance to the stenosis. The stenoses were $3 \mathrm{~mm}$ in overall length and tapered with a $45^{\circ}$ angle on the ends, and their cross-sectional area reductions were $50 \%, 75 \%$ and $90 \%$. The model and the stenoses were cast from $\rho c$ rubber (PRC 1538, Product Research and Chemical Corp., Glendale, CA). The speed of sound of this material and of various mixtures of glycerol and water were measured from the time required for a sinusoidal burst to traverse a known distance. The percent mismatch in acoustic impedance between the model and the fluid was calculated as $200\left(\rho_{\mathrm{r}} \mathbf{c}_{\mathrm{r}}-\rho_{\mathrm{f}} \mathbf{c}_{\mathrm{f}}\right) /\left(\rho_{\mathrm{r}} \mathbf{c}_{\mathrm{r}}+\rho_{\mathrm{f}} \mathbf{c}_{\mathrm{f}}\right)$, where $\rho$ is density, $c$ is speed of sound, and the subscripts $r$ and $f$ correspond to the rubber and the fluid, respectively. These values are shown along with kinematic viscosities in Table 1. The fluid was seeded with corn starch to provide scatterers for the sound. These particles were found, from micrographs, to range between 5 and 20 $\mu \mathrm{m}$ in diameter.

The experimental setup is shown in Fig. 1. Fluid was driven by gravity from the upstream constanthead reservoir through the test section and into the downstream reservoir. It was returned to the upstream reservoir through a centrifugal pump. Flow rate was controlled by the height of the reservoir and the setting of the valve between the constant head tank and the model. The test section was mounted so that flow was in the direction of gravity to prevent the seeding particles from settling laterally in the test section. The surface tension at the bottom of the model was sufficient to stabilize the meniscus and keep the fluid attached to the model at the exit. The criterion for this is given by Michael $(1981)$ as $r \sqrt{\mathrm{p}_{\mathrm{f}} \mathrm{g} / \gamma}<2.404$, where $\mathrm{r}$ is tube radius, $\rho_{\mathrm{f}}$ is fluid density, $\mathrm{g}$ is acceleration due to gravity and $\gamma$ is the surface tension. For these experiments $\mathrm{r} \sqrt{\rho_{\mathrm{f}} \mathrm{g} / \gamma}=0.62$. The flow rate was calibrated from the time required to collect a given volume of fluid from the model exit. Two steady flow Reynolds numbers, 240 and 400, were used, which correspond, respectively, to the mean and peak values in the coronary artery. Reynolds numbers were based on the unstenosed tube diameter, $d$, and cross-sectional average velocity in the unstenosed part of the tube, $u_{a v}$. For 
Table 1. Properties of the fluid and the model.

\begin{tabular}{lccccc}
\hline $\begin{array}{c}\text { Glycerol } \\
\text { (\% by weight) }\end{array}$ & $\begin{array}{c}\text { Mismatch } \\
(\%)\end{array}$ & $\begin{array}{c}\mathrm{c}_{\mathrm{f}} \\
(\mathrm{cm} / \mathrm{s})\end{array}$ & $\begin{array}{c}\mathrm{p}_{\mathrm{f}} \\
\left(\mathrm{g} / \mathrm{cm}^{3}\right)\end{array}$ & $\begin{array}{c}\mathrm{p}_{\mathrm{f}} \mathrm{c}_{\mathrm{f}} \\
\left(\mathrm{g} \mathrm{cm}^{-2} \mathrm{~s}^{-2}\right)\end{array}$ & $\begin{array}{c}\nu \\
\left(\mathrm{cm}^{2} / \mathrm{s}\right)\end{array}$ \\
\hline 20 & 0 & 155,000 & 1.04 & 161,000 & 0.017 \\
0 & 7 & 150,000 & 1.00 & 150,000 & 0.0091 \\
40 & -12 & 168,000 & 1.08 & 181,000 & 0.035 \\
PRC Rubber & - & 154,000 & 1.05 & 162,000 & - \\
\hline
\end{tabular}

convenience, $u_{p}$ is used for the theoretical maximum for Poiseuille flow in the $3.18-\mathrm{mm}$ tube, and $u_{\mathrm{s}}$ is the cross-sectional average velocity at the throat of the stenosis. Similarly, $f_{a v}, f_{p}$ and $f_{s}$ are the Doppler frequencies that correspond to $\mathrm{u}_{\mathrm{av}}, \mathrm{u}_{\mathrm{p}}$ and $\mathrm{u}_{\mathrm{s}}$, respectively.

For the Poiseuille flow experiments, the guidewire was inserted into the model from the downstream end, and mounted on the centerline of the flow. For the stenosis experiments, the guidewire was inserted through a hole in the side of the model, as shown in Fig. 1. This latter configuration imitates the in vivo measurement method, where precise lateral positioning of the guidewire is not possible. The tip of the catheter was placed $3 \mathrm{~mm}$ from the upstream end of the stenosis with the aid of a centimeter scale. The error in axial placement is estimated to be $\pm 0.5 \mathrm{~mm}$. The range gate was varied such that measurements $2.60,3.77,4.94$, $5.20,6.24,7.41,8.58$ and $9.75 \mathrm{~mm}$ from the catheter tip were taken. The first measurement is upstream of the stenosis, the next three are inside the stenosis, and the last four are downstream of the stenosis. It was desirable to position the guidewire on the centerline of the tube so that the sample volume would include as much of the vessel cross-section as possible. Therefore, the guidewire was manipulated until the highestpitched sound was obtained.

The Cardiometrics guidewire used in this study was $175-\mathrm{cm}$ long and $0.457 \mathrm{~mm}(0.018$ inch $)$ in diameter. It was operated at a pulse repetition frequency of $67.4 \mathrm{kHz}$. The quadrature signals from the electronics were filtered and then digitized on a Masscomp 5500 (Concurrent Computer Corp., Richardson, TX) data acquisition computer. The sample frequency was either 40,60 or $80 \mathrm{kHz}$, whichever was just high enough to capture the expected frequency content based on the highest flow velocities for the given flow conditions. Data were then processed by FFT-based power spectral analysis. The low pass filter (Krohn-Hite 3343, Cambridge, MA) was a dual channel eight pole butterworth, and the cutoff was set to $\frac{3}{8}$ the sampling frequency for the $50 \%$ and $75 \%$ stenoses. As a result of sampling rate limitations, the cutoff was set to $\frac{5}{8}$ the sampling rate for the $90 \%$ stenosis to prevent distortion of the high frequency peaks.

Prior to the flow measurements, the sample vol- ume of the guidewire was measured with a stringtarget setup. The beam direction was perpendicular to the constant string velocity, and the position of the guidewire was varied in a direction perpendicular to both the beam direction and the string velocity. Thus, the quadrature signals resulted from transit time broadening effects of the target. The quadrature signals were digitized and their root-mean-square power was computed as a function of position. This was repeated for various range gate settings.

\section{RESULTS}

\section{Sample volume measurements}

Figure 2 shows the sample volume width measurements for ranges of 2.6, 5.2 and $10.0 \mathrm{~mm}$. The distance between the locations where power is half the peak value increases with range from $1 \mathrm{~mm}$ at $2.6 \mathrm{~mm}$ to $1.7 \mathrm{~mm}$ at $5.2 \mathrm{~mm}$ to $3.1 \mathrm{~mm}$ at $10.0 \mathrm{~mm}$. Thus, only at the farthest range does the sample volume (as defined by this arbitrary measure) intersect the entire lumen. Even then, it must be remembered that the

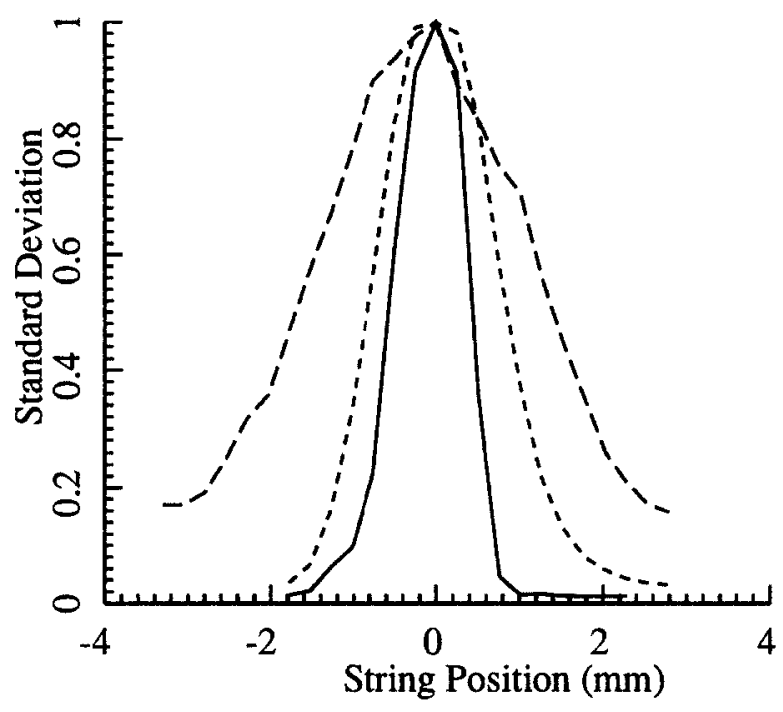

Fig. 2. Beam patterns for the Cardiometrics guidewire at various ranges as measured by the string target system. The value used in the ordinate is the standard deviation of the voltage from the quadrature signals normalized such that the largest value is 1 . 
beam pattern is not uniform over the lumen area. The depth (thickness) of the sample volume was measured by the same technique and found to be $1.3 \mathrm{~mm}, 1.15$ $\mathrm{mm}$ and $1.0 \mathrm{~mm}$ at ranges of $2.5 \mathrm{~mm}, 5.2 \mathrm{~mm}$ and $10.0 \mathrm{~mm}$, respectively.

\section{Poiseuille flow measurements}

Power spectra from the unobstructed straight tube for ranges of $2.6 \mathrm{~mm}, 5.2 \mathrm{~mm}$ and $10 \mathrm{~mm}$ show strong spectral distortion if the acoustic impedances of fluid and model material are not matched (Fig. 3). The expected Doppler shifts from the centerline velocity are shown as vertical lines above the spectra. The velocities for the $12 \%, 7 \%$ and $0 \%$ mismatch cases were $23 \mathrm{~cm} / \mathrm{s}, 26.7 \mathrm{~cm} / \mathrm{s}$ and $20.5 \mathrm{~cm} / \mathrm{s}$, respectively. Since kinematic viscosity differed for the three fluids, the Reynolds numbers differed and ranged from 185 (12\% mismatch) to $800(0 \%$ mismatch $)$. However, the Reynolds numbers were well below 2000 and the entry length was $>0.08 \mathrm{ReD}+0.7$ so that Poiseuille flow was assured (White 1974). For comparison, the spec- trum, based on the velocity profile and the beam pattern at a range of $5.2 \mathrm{~mm}$, was computed numerically and is shown in Fig. 3d. For mismatches of $12 \%$ and $7 \%$. substantial spectral content is present for all ranges at frequencies which are significantly lower than $\mathrm{f}_{p}(e . g$. between 0 and $2 f_{p} / 3$ ). The power associated with the Doppler frequency decreases with increased mismatch. so that a more uniform spectrum occurs, which may have distinct peaks in the lower frequency range. A low frequency peak in the spectrum with $12 \%$ mismatch and range $10 \mathrm{~mm}$ surpasses the peak near $f_{p}$ in amplitude. At this range, even the lowest mismatch case (Fig. 3c) exhibits two low-level peaks in the lower frequency range.

\section{Stenosis measurements, $0 \%$ mismatch}

$50 \%$ Area reduction. Figure 4 shows power spectra for the $50 \%$ area reduction at Reynolds number 240. The axial locations are $0.4 \mathrm{~mm}$ upstream of the stenosis inlet, $1.94 \mathrm{~mm}$ into the stenosis (i.e., $1.94 \mathrm{~mm}$
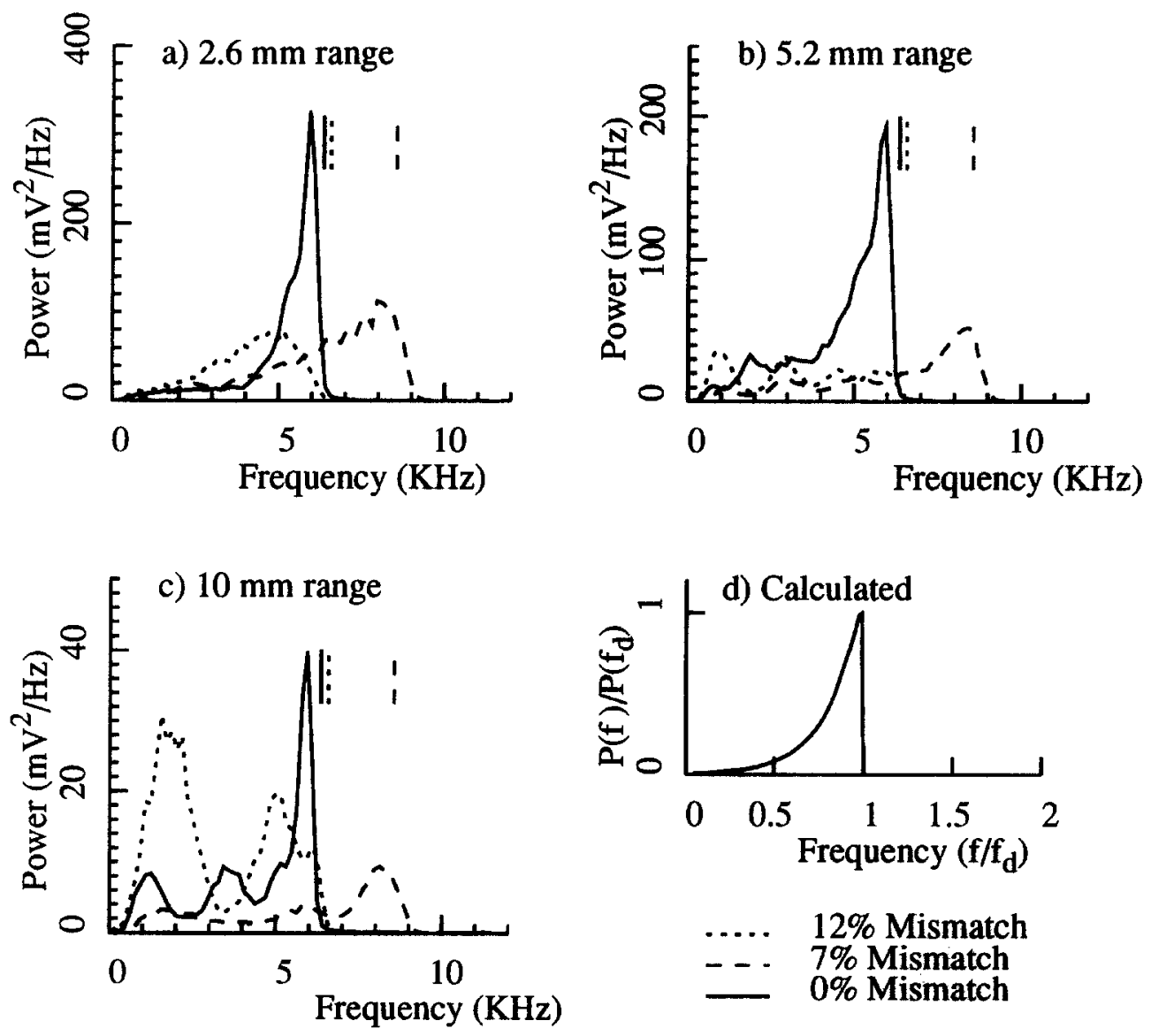

Fig. 3. Doppler power spectra from laminar flow in a straight 3.18-mm tube with various acoustic impedance mismatches and at three different ranges. The vertical lines above the graphs indicate the frequency $f_{p}$ for the maximum of Poiseuille flow for the mismatch associated with that line mode. 

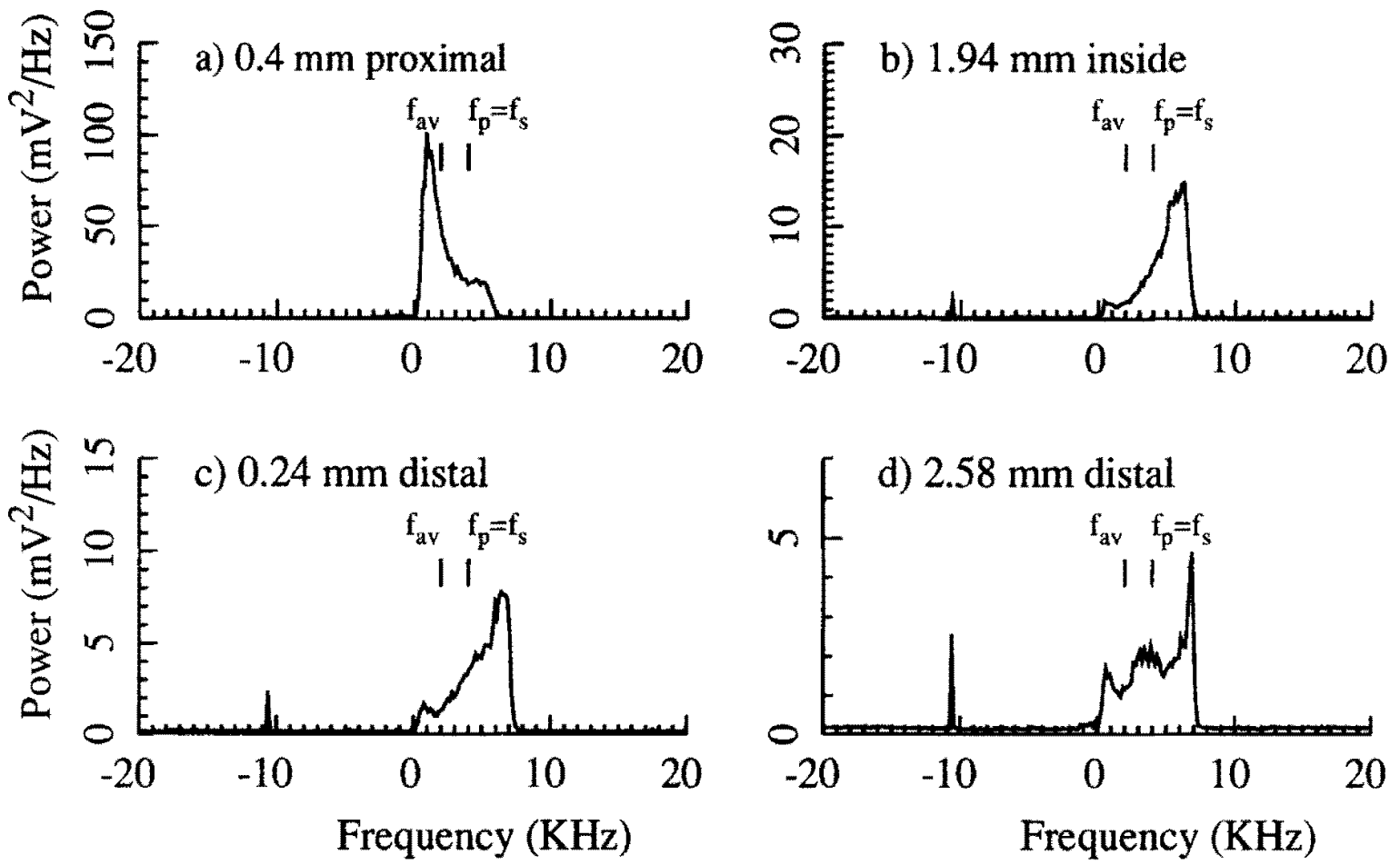

Fig. 4. Doppler power spectra from the $50 \%$ area reduction model with $0 \%$ acoustic impedance mismatch at Reynolds number 240 . The frequencies $f_{a v}, f_{p}$ and $f_{s}$ are indicated by the vertical bars above the spectra.

distal to the stenosis inlet), $0.24 \mathrm{~mm}$ downstream of the stenosis exit, and $2.58 \mathrm{~mm}$ downstream of the stenosis exit.

The spike at $-10 \mathrm{kHz}$ in these spectra was noise from the guidewire instrumentation. It was present for other flow conditions, including zero flow. The proximal spectrum exhibits a higher proportion of low frequency power than would be expected from a uniform beam pattern in Poiseuille flow. This is expected since the bearn half-power width is significantly narrower than the artery at this range, and since the catheter is near the wall, so a large amount of sound is scattered from the lower velocity fluid at this radial position. The maximum frequency in this spectrum is near $6 \mathrm{kHz}$. The theoretical maximum velocity for Poiseuille flow is $u_{p}=27 \mathrm{~cm} / \mathrm{s}$, which corresponds to a Doppler shift of $f_{p}=4.18 \mathrm{kHz}$. This implies that the velocity profile may be influenced by the stenosis, which is reasonable since the sample volume is long enough $(1 \mathrm{~mm})$ that part of it may be inside the stenosis.

Within the stenosis, the power density is concentrated at the higher frequency end of the spectrum. The peak is at $6.2 \mathrm{kHz}$, so that the ratio of this frequency to $f_{s}$ is 1.5 , which indicates a partially-developed profile. The spectrum $0.24 \mathrm{~mm}$ downstream from the stenosis exit exhibits a similar shape. The ratio of the peak frequency to $f_{s}$ is increased to 1.67 , which is explained by the further development of the velocity profile within the stenosis.

At $2.58 \mathrm{~mm}$ distal to the stenosis, three distinct regions are seen in the power spectrum. The peak at $7 \mathrm{kHz}$ corresponds to the exit jet seen in the previous two spectra. The peak at $1 \mathrm{kHz}$ can be attributed to the forward flow region of the separation zone. The origin of the broad peak between 1.5 and $5 \mathrm{kHz}$ cannot be determined without detailed velocity profile measurements in the model, but velocities of this magnitude must arise from the region between the recirculation zone and the jet.

Figure 5 shows power spectra $0.4 \mathrm{~mm}$ upstream, $1.94 \mathrm{~mm}$ into, $0.24 \mathrm{~mm}$ distal to and $2.58 \mathrm{~mm}$ distal to the $50 \%$ area reduction stenosis for Reynolds number 400 . The frequencies $f_{a v}, f_{p}$ and $f_{s}$ are, respectively, $3.5 \mathrm{kHz}, 7.0 \mathrm{kHz}$ and $7.0 \mathrm{kHz}$. The spectra generally show features similar to the Reynolds number 240 case. The upstream spectrum (Fig. 5a) is skewed toward the low frequency region, with most of the power density at frequencies less than $f_{p}$. The spectrum from inside the stenosis (Fig. 5b) shows a sharp high frequency peak at $10 \mathrm{kHz}$, which corresponds to a nearly blunt flow with a maximum of 1.43 times $u_{s}$. The same spectral shape is seen $0.24 \mathrm{~mm}$ distal to the stenosis, except that the main peak is slightly higher in frequency, which may reflect a higher centerline velocity 

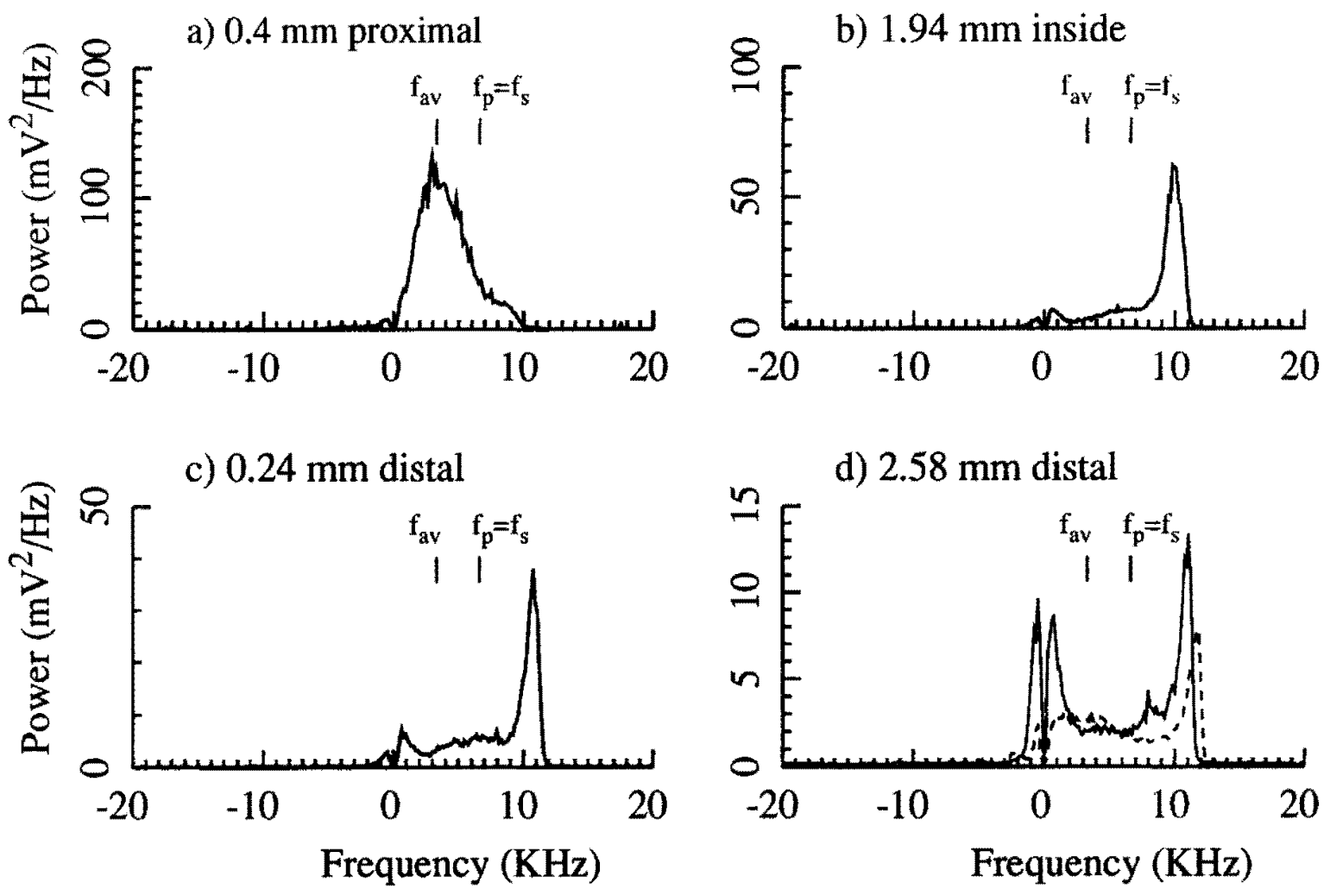

Fig. 5. Doppler power spectra from the $50 \%$ area reduction model with $0 \%$ acoustic impedance mismatch at Reynolds number 400 . The dashed line in (d) is a second realization under the same flow conditions as the solid line.
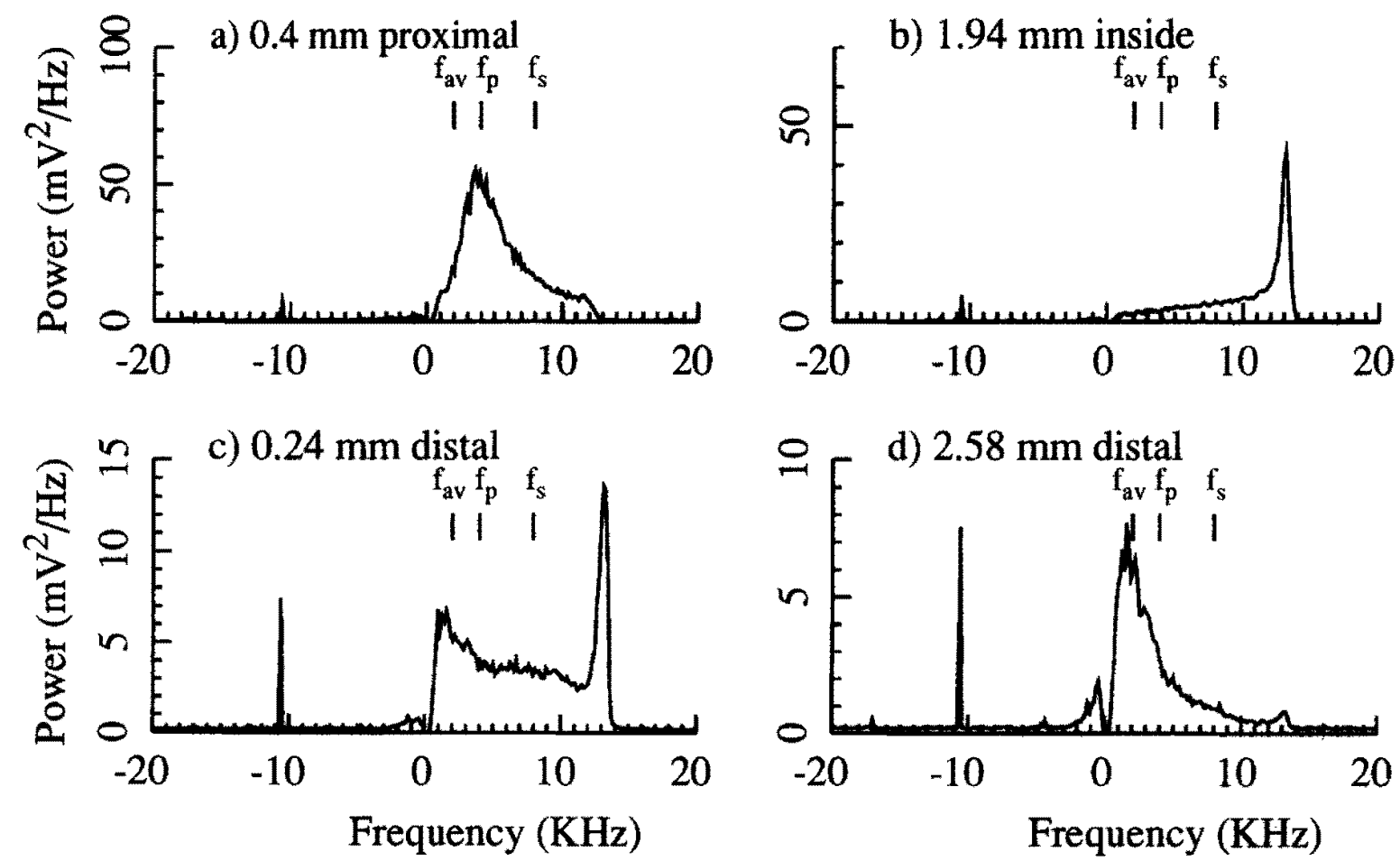

Fig. 6. Doppler power spectra from the $75 \%$ area reduction model with $0 \%$ acoustic impedance mismatch at Reynolds number 240 . 


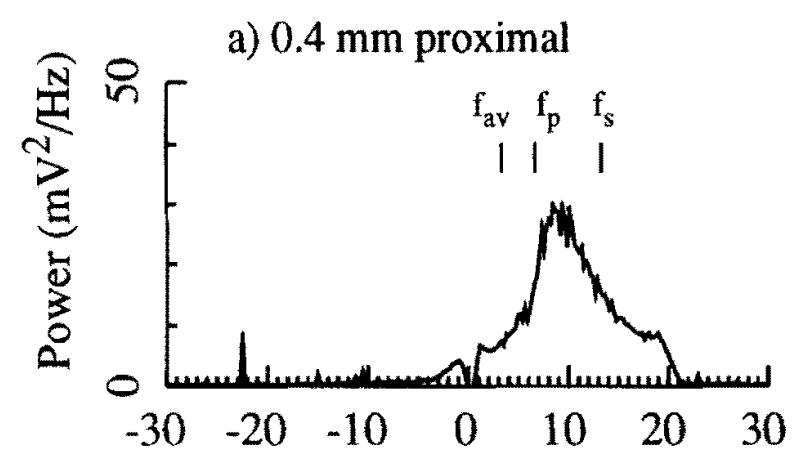

c) $0.24 \mathrm{~mm}$ distal

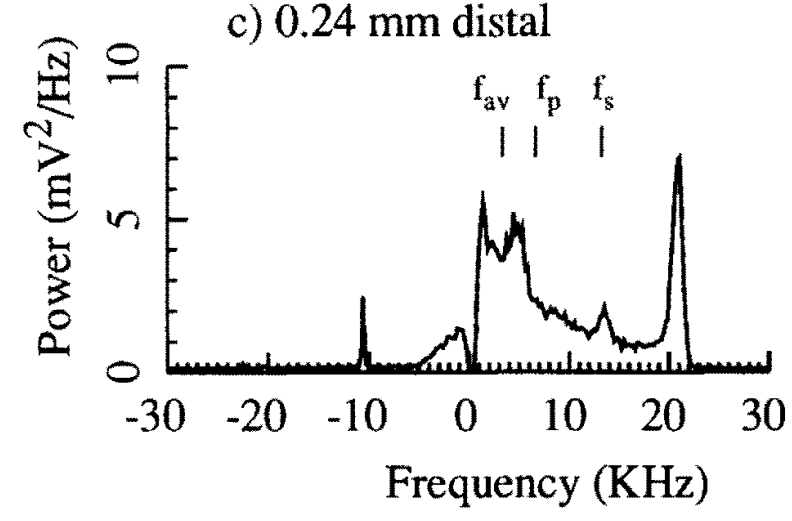

b) $1.94 \mathrm{~mm}$ inside

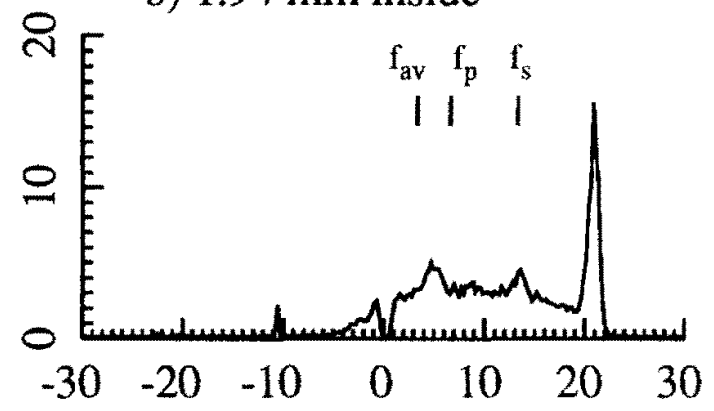

d) $2.58 \mathrm{~mm}$ distal

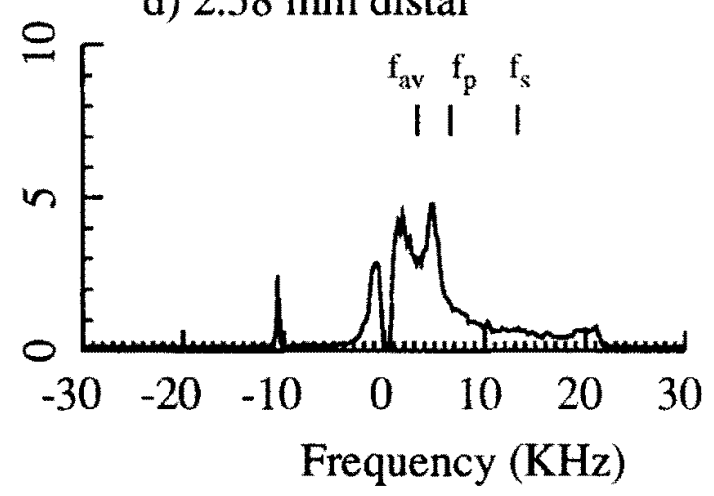

Fig. 7. Doppler power spectra from the $75 \%$ area reduction model with $0 \%$ acoustic impedance mismatch at Reynolds number 400 .

as a result of profile development. At a range 2.58 $\mathrm{mm}$ distal to the stenosis, the peak associated with the emerging jet is still visible, and two exceptionally strong peaks, which can be attributed to the forward and reverse flow in the recirculation region, are seen at low frequency. Although the two data sets in Fig. $5 \mathrm{~d}$ were taken under identical conditions, the spectra are not identical in that the dashed line exhibits minimal forward or reverse recirculation. Although guidewire placement could explain this, an alternative explanation is a change in the flow patterns themselves. At Reynolds numbers that correspond to transitional flow, low level background vibration noise can cause a shift in the location at which instabilities occur (Jones and Fronek 1988), and this can alter the flow patterns at a given location.

$75 \%$ Area reduction. Power spectra for the $75 \%$ area reduction stenosis at a Reynolds number of 240 are shown in Fig. 6. The axial locations are $0.4 \mathrm{~mm}$ proximal to, $1.94 \mathrm{~mm}$ into, $0.24 \mathrm{~mm}$ distal to, and $2.58 \mathrm{~mm}$ distal to the stenosis. The first three spectra are similar in character to those for the $50 \%$ area reduction at similar axial locations. The frequencies $f_{a v}, f_{p}$ and $f_{s}$ are $2.1 \mathrm{kHz}, 4.2$ $\mathrm{kHz}$ and $8.4 \mathrm{kHz}$, respectively. The frequency of the peak which corresponds to the exit jet is at $12.8 \mathrm{kHz}$, or 1.52 times $\mathrm{f}_{\mathrm{s}}$. This peak, though still present $2.58 \mathrm{~mm}$ distal, is reduced dramatically in power. It is proposed that this occurs because the flow has become turbulent, with a stable transition between 0.24 and $2.58 \mathrm{~mm}$, so that the high velocity jet has dissipated.

Results at Reynolds number 400 are similar to those at Reynolds number 240 , as can be seen from the spectra taken $0.4 \mathrm{~mm}$ proximal to, $1.94 \mathrm{~mm}$ into, $0.24 \mathrm{~mm}$ distal to, and $2.58 \mathrm{~mm}$ distal to the stenosis (Fig. 7). The landmark frequencies for this case are $f_{a v}=3.48 \mathrm{kHz}, f_{p}=6.97 \mathrm{kHz}$ and $f_{s}=13.9 \mathrm{kHz}$. The jet velocity is exhibited as a peak at 1.45 times $f_{s}$. Again, the sharp peaks at -10 and $-22 \mathrm{kHz}$ represent instrumentation noise.

90\% Area reduction. Measurements on this constriction were limited to Reynolds number 240 , partly because aliasing was problematic when the jet velocity became too high, and partly because the pressure head required to drive the flow through the tight constriction was substantial. Spectra are shown in Fig. 8 for axial positions $0.4 \mathrm{~mm}$ proximal to, $1.94 \mathrm{~mm}$ into, $0.24 \mathrm{~mm}$ distal to and $2.58 \mathrm{~mm}$ distal to the stenosis. If the peaks at low negative frequencies (ca. $33 \mathrm{kHz}$ ) are ignored, the results are similar to those discussed above. The jet peak is at $33 \mathrm{kHz}$, or $1.57 \mathrm{f}_{\mathrm{s}}$. Here, $\mathrm{f}_{\mathrm{av}}$ is $2.09 \mathrm{kHz}$, 

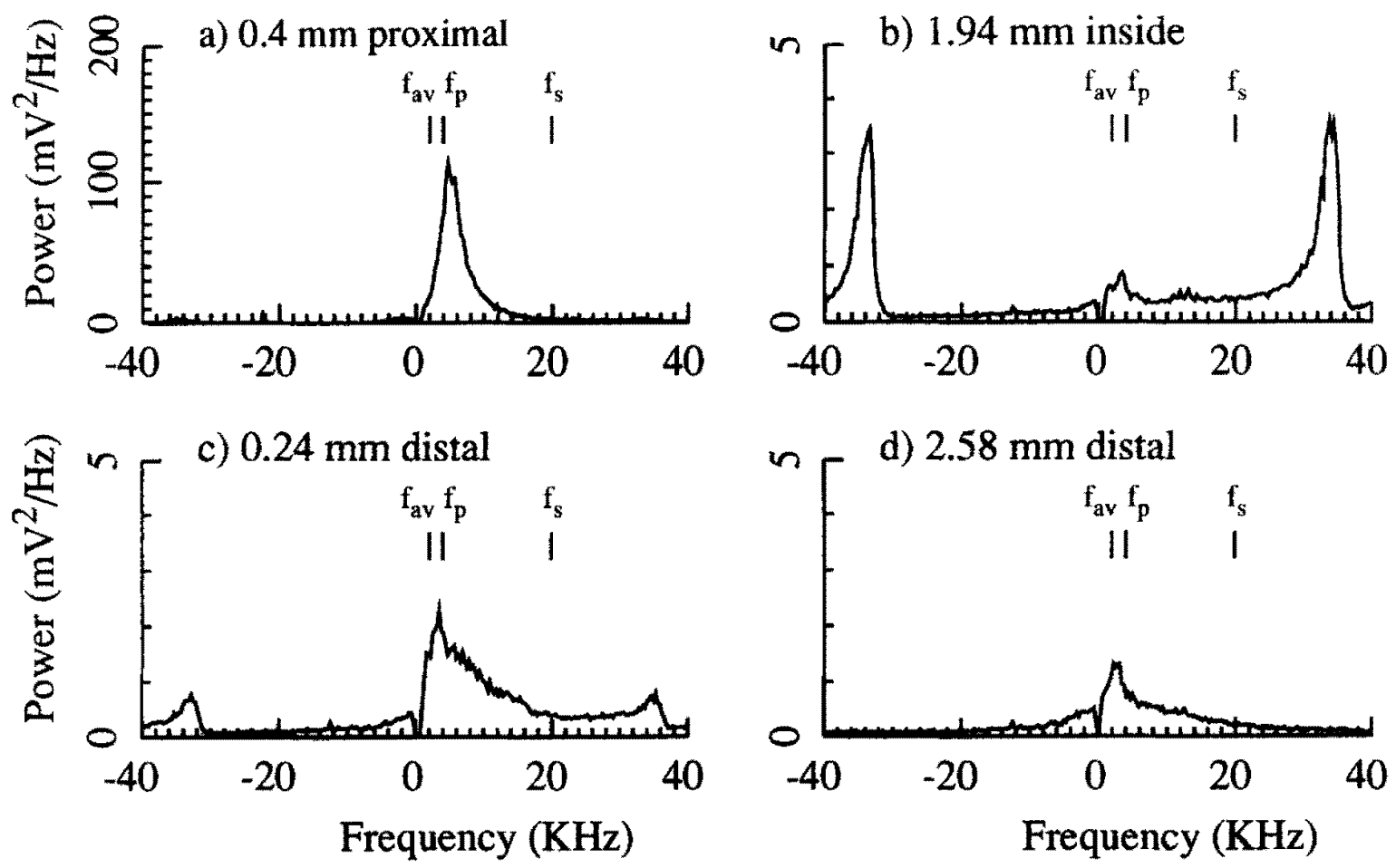

Fig. 8. Doppler power spectra from the $90 \%$ area reduction model with $0 \%$ acoustic impedance mismatch at Reynolds number 240 .

$f_{p}$ is $4.18 \mathrm{kHz}$, and $f_{s}$ is $20.9 \mathrm{kHz}$. The decay of the jet velocity peak occurs much closer to the stenosis than in the previous case. This is consistent with the previous observations that turbulent transition occurs closer to the stenosis as the Reynolds number and stenosis severity increase (Back and Roschke 1972). The peak at high negative frequency is a result of aliasing, as evidenced through its shift from the jet exit frequency by the pulse repetition frequency.

\section{Stenosis measurements, $7 \%$ mismatch}

The differences between the $7 \%$ mismatch case and the $0 \%$ mismatch case with a stenosis present are not as readily defined as they were in the case of no stenosis. For the $50 \%$ area reduction, two differences were seen between the shapes of the $7 \%$ mismatch spectra and the $0 \%$ mismatch spectra for the same stenosis and Reynolds number. First, in the throat of the stenosis (Fig. 9a compared to Fig. 4b, and Fig. 9c compared to Fig. 5b), the spectrum did not consist solely of the high frequency peak indicative of blunt flow. There was substantial low frequency content at $7 \%$ mismatch, which has no immediate hemodynamic explanation. Secondly, there was no significant power in the low-negative frequency part of the spectrum downstream of the stenosis for the Reynolds number 400 case (Fig. 9d compared to Fig. 5d). The absence of reverse flow can be explained to some extent by the high pass filtering of the quadrature signals. Since the kinematic viscosity of water is less than that of the fluid used for the $0 \%$ mismatch case, the velocities are lower for a given Reynolds number by a factor of 0.54 . The high pass filter is thus expected to remove more of the low frequency components. However, the total absence of reverse flow seen in Figs. $9 \mathrm{~b}$ and $9 \mathrm{~d}$ is too dramatic to be accounted for by this effect.

A further feature of the $50 \%$ area reduction, $7 \%$ mismatch spectra is the prominence of the peaks, particularly in Figs. $9 \mathrm{~b}$ and $9 \mathrm{~d}$. In contrast, the $0 \%$ mismatch spectra show relatively smooth transitions between peaks and valleys.

The $7 \%$ mismatch spectra for the $75 \%$ area reduction (Fig. 10) are similar to the corresponding spectra for the $0 \%$ mismatch case under hemodynamically similar conditions (Figs. 6 and 7). For Reynolds number 240 , the throat region (Fig. 10a) generates a prominent high frequency peak, but the spectrum has more low-positive frequency power than does the $0 \%$ mismatch spectrum. The spectrum from further downstream (Fig. 10b) has power at low-negative frequency, a low-positive frequency peak and a small-high frequency peak, which is similar to the case with $0 \%$ mismatch. For Reynolds number 400 , there are no hemodynamically-related features that distinguish the 

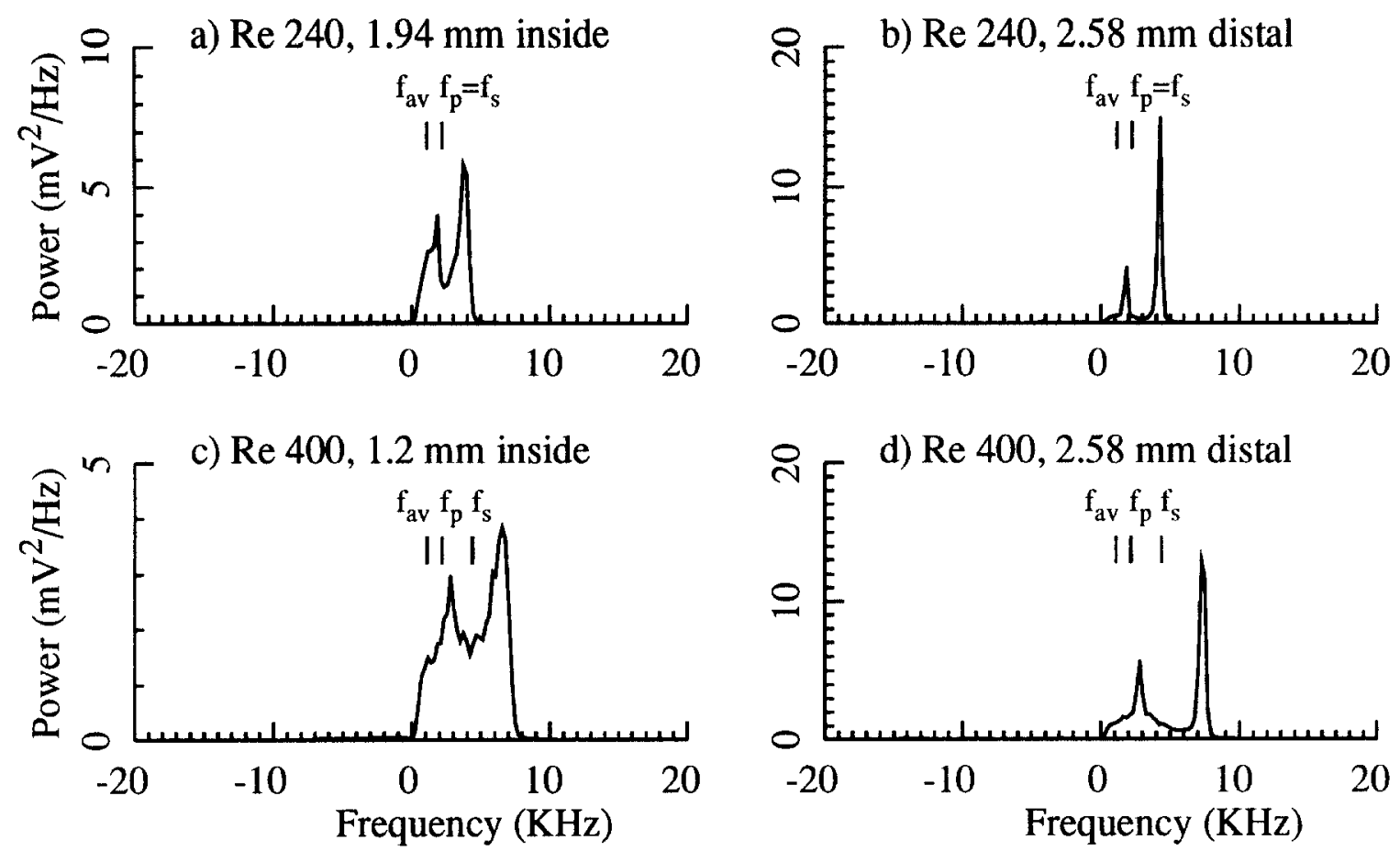

Fig. 9. Doppler power spectra from the $50 \%$ area reduction model with $7 \%$ acoustic impedance mismatch.

$0 \%$ and $7 \%$ mismatch spectra, either at the throat (Fig. $10 \mathrm{c}$ ) or distal to the stenosis (Fig. 10d).

When the frequency of the peak near $f_{s}$ is divided by $\mathrm{f}_{\mathrm{s}}$ for positions $1.94 \mathrm{~mm}$ and $2.2 \mathrm{~mm}$ into the stenosis and for the position $0.24 \mathrm{~mm}$ distal, the ratios are generally near 1.5 for the data with both $0 \%$ and $7 \%$
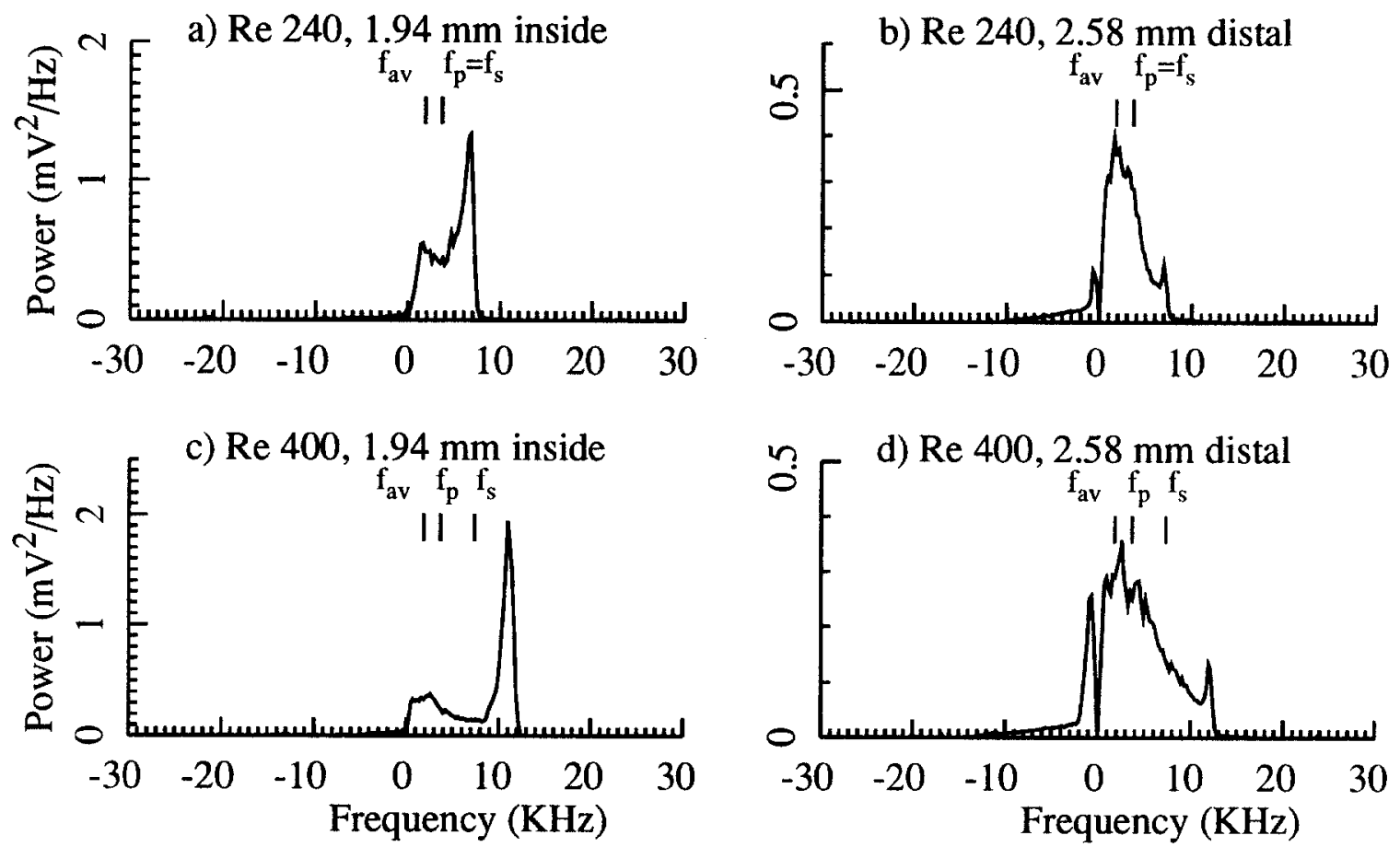

Fig. 10. Doppler power spectra from the $75 \%$ area reduction model with $7 \%$ acoustic impedance mismatch. 
mismatch. The average value for the $7 \%$ mismatch case was 1.65 , which was higher than the value of 1.54 found for the $0 \%$ mismatch case. The difference in ratios for the two mismatches was shown to be significant at the level of $p<0.01$ by a paired Student's ttest. Also, the standard deviation for the $7 \%$ mismatch case was a factor of two higher than that for the $0 \%$ mismatch case.

\section{DISCUSSION}

Although the power spectrum for an uniform beam pattern in Poiseuille flow has constant power in the frequency range from 0 to $f_{p}$, the spectra shown in Fig. 3 for the zero mismatch case have a prominent peak near $f_{p}$. This is consistent with the probe beam pattern, which has most of its power concentrated near the higher velocity fluid on the tube centerline. Thus, the calculated spectrum of Fig. $3 \mathrm{~d}$ and the power spectral shapes obtained with zero mismatch are reasonable.

In previous in vitro investigations on Doppler ultrasound, the mismatch in acoustic impedance between the model wall and the blood analogue fluid has been of practical importance. Whenever a mismatch interface exists, the amount of sound transmitted through it is reduced. The wall and fluid must be sufficiently well matched to allow an acceptable signal return at the receiver or measurement accuracy will be compromised. Another effect, discussed by Thompson et al. (1990), is an alteration in the beam pattern caused by total reflection of parts of the beam. This can occur because the propagation angles of all the wavefronts from a probe are not equal. In the case discussed here, however, the sound is not transmitted through a barrier, but rather confined within a cylindrical geometry. It is this geometry that determines the acoustic boundary conditions for the probe beam pattern and distorts it from the shape it would have in an unconfined region. It is proposed here that the low frequency peaks in Figs. $3 \mathrm{a}, 3 \mathrm{~b}$ and $3 \mathrm{c}$ are caused by such a distortion. Specifically, the reflections from the fluid-model interface may cause regions of high beam intensity at radial positions off centerline in the tube. The reflections may also change the local propagation direction of sound. which can also alter the Doppler spectrum (Newhouse et al. 1980). These latter effects should be small, however, since the flow is para-axial and since the spreading angle of the beam is small.

The increased spectral distortion with increasing range is evidence that this distortion is caused by interaction between the beam pattern and the wall interface. As depth increases, the beam widens, as shown in Fig. 2 , so the beam-wall interaction must increase as well.

The $7 \%$ and $12 \%$ mismatches differ from one another not only in magnitude, but also in sign. For $7 \%$, the fluid has a lower sound speed than the model, whereas for $12 \%$ the fluid has a higher sound speed. Although both cases cause distorted spectral shapes, the 7\% mismatch case may be further complicated by total internal reflection of part of the ultrasound beam from the wall.

Since the walls of the model used in these studies were approximately 1 inch $(25.4 \mathrm{~mm})$ thick, and since the probe beam patterns were narrow, the acoustic interface between the model and the surrounding air was sufficiently far from the transmitted sound to have negligible mismatch effects. In vivo, the acoustic mismatch between the external wall of the artery and the surrounding tissue can either enhance or partially cancel these effects.

The acoustic impedance of blood at $40 \%$ hematocrit is $1.64 \times 10^{5} \mathrm{~g} \mathrm{~cm}^{-2} \mathrm{~s}^{-1}$ (Goss et al. 1978), and Table 2 shows the acoustic impedance for aortic tissues under various conditions as compiled by Greenleaf et al. (1974). The value for normal tissue is $1.56 \times 10^{5}$ $\mathrm{g} \mathrm{cm}^{-2} \mathrm{~s}^{-1}$, which indicates a mismatch of $6.8 \%$. As the artery becomes more fibrous, the acoustic impedance mismatch decreases at first, which implies that the Doppler signals will improve. As the artery becomes calcified, however, the mismatch increases again. If the impedances of the abdominal aorta and the coronary artery are similar, in vivo coronary Doppler ultrasound spectra may exhibit significant distortion.

The data exhibit specific patterns which can be directly related to the fluid mechanics of the stenosis. Upstream of the stenosis, the power is concentrated at frequencies near $f_{a v}$, with some velocities near $f_{s}$ caused by a portion of the sample volume which is inside the stenosis throat. In the throat, a prominent peak exists between $\mathrm{f}_{\mathrm{s}}$, and $2 \mathrm{f}_{\mathrm{s}}$, and there is minimal spectral content at other frequencies. Distal to the stenosis, there are up to three prominent peaks. One is between $f_{s}$ and $2 f_{s}$ and corresponds to the jet velocity, another is in the low-positive frequencies and corresponds to the forward part of the flow recirculation region, and the other is in the lownegative frequencies and corresponds to reverse flow. Further distal, the peak near $f_{s}$ is attenuated if the Reyn-

Table 2. In vivo acoustic impedances for abdominal aorta.

\begin{tabular}{lcc}
\hline \multicolumn{1}{c}{ Type } & $\begin{array}{c}\text { Impedance } \\
\mathrm{g} /\left(\mathrm{cm}^{2} \mathrm{~s}\right)\end{array}$ & Mismatch $(\%)$ \\
\hline Normal & 156,000 & $-5.0(-6.9$ to -3.7$)$ \\
Fibrous & 165,000 & $+0.6(-5.6$ to +3.6$)$ \\
Fatty & 169,000 & $+3.0(-1.2$ to +12.6$)$ \\
Calcified & 176,000 & $+7.0(+5.9$ to +8.2$)$ \\
Fresh whole blood $\dagger$ & 164,000 & \\
\hline
\end{tabular}

† From Kikuchi et al., 1972. 
olds number is sufficiently high, and this is consistent with the decrease in jet velocity associated with transition to turbulence.

The $7 \%$ mismatch data were less consistent than the $0 \%$ mismatch data. They exhibited low frequency spectral content at the stenosis throat for both the $50 \%$ and $75 \%$ area reduction. Also, for the $50 \%$ area reduction, the spectral peaks were more prominent with the higher mismatch. Other spectral distortions may exist, but cannot be determined without more quantitative measurements of the velocity field and more precise localization of the sample volume.

Although the $0 \%$ and $7 \%$ mismatch cases were hemodynamically similar in that their Reynolds numbers and stenotic area reductions were identical, they were different in some of the parameters that affect the Doppler measurement. There was a slight difference in the positions at which data were taken for the $0 \%$ and $7 \%$ mismatch data since the sound speeds differed by approximately $3.3 \%$. This corresponded to a shift of approximately $0.2 \mathrm{~mm}$ at the exit of the constriction. Also, the absence of reverse flow in the $7 \%$ mismatch case may result because the flow velocities are lower for the same Reynolds number and thus the frequency range for the spectrum is smaller. The high pass filter thus filters out a higher percentage of this reduced frequency range. However, the reduced velocities in the $7 \%$ mismatch case should not affect the percent of spectral broadening caused by ambiguity since this broadening is proportional to velocity (Garbini et al. 1982).

Two aspects of intracoronary Doppler diagnosis make the clinical application of these results difficult. First, the results depend to some extent, on the placement of the guidewire. Currently no method is available to provide accurate placement of the sample volume in vivo. Secondly, the spectra shown here were averaged over $1.47 \mathrm{~s}$. To obtain spectra of comparable stability from in vivo measurements, ensemble averaging from a number of cardiac cycles on the order of 100 is required.

The in vitro model was substantially idealized in comparison with clinical measurements. In vivo, pulsatile flow, shorter entrance lengths and curvature combine so that the velocity profiles are not parabolic and wall motions result from pressure fluctuations. However, Ahmed and Giddens (1984) have shown that the reverse flow velocities and degree of recirculation distal to a stenosis are similar in pulsatile and steady flow, and that both conditions exhibit blunt velocity profiles at the throat of the constriction. It has been shown (Latornell and Pollard 1986) that the upstream velocity profile affects the stability of the post-stenotic flow and hence the length of the reattachment region. Thus, it is not possible from the results presented here to predict the amount of reverse flow expected from a given degree of stenosis in vivo. The primary effect of wall motion is the artifact it induces on the low frequency part of the Doppler spectrum, and this could severely hinder the detection of velocities in the reverse flow region.

\section{CONCLUSIONS}

Acoustic impedance mismatch can strongly affect intravascular Doppler ultrasound measurements, and the typical mismatches between blood and arterial walls in vivo are of sufficient magnitude to be affected by this phenomenon. With a mismatch of $0 \%$ between the blood and the arterial wall, ensembled Doppler ultrasound spectra from the guidewire can be interpreted in terms of the fluid mechanics of post-stenotic flow. Within the throat of the stenosis, a peak is seen near a value of 1.5 times the frequency, which corresponds to the cross-sectional average velocity within the throat of the stenosis. Downstream of the stenosis, peaks are seen that correspond to the emerging jet and the forward and reverse flow portions of the recirculation region. The peak which corresponds to the jet attenuates rapidly with axial distance when Reynolds numbers are sufficiently high to result in distal turbulent flow.

With $7 \%$ mismatch, the features seen with $0 \%$ mismatch were present in some cases, but the results were not consistent. Specifically, the $50 \%$ area reduction resulted in an additional low-frequency peak within the stenosis throat and an absence of detected reverse flow distal to the stenosis. To accurately interpret the power spectra of the intracoronary device in terms of the velocity profiles, it is necessary to understand the acoustic environment in which the measurements are made, as well as the effect this environment has on the returned Doppler signals.

\section{REFERENCES}

Ahmed SA, Giddens DP. Pulsatile poststenotic flow studies with laser Doppler anemometry. I Biomech 1984;17:695-70S.

Back LH, Roschke EJ. Shear-layer flow regimes and wave instabilities and reattachment lengths downstream of an abrupt circular channel expansion. I Appl Mech 1972;39:677 681 .

Bassiouny $H S$, White $S$, Glagov $S$, et al. Anastomotic intimal hyperplasia: mechanical injury or flow induced. I Vase Surg 1992; $15: 708-716$.

Chesebro JH, Zoldhelyi P, Fuster V. Pathogenesis of thrombosis in unstable angina. Am $\mathrm{J}$ Cardiol $1991 ; 68: 2 \mathrm{~B}-10 \mathrm{~B}$.

Dintenfass L. Rheological approach to thrombosis and atheroselerosis. Angiology $1964 ; 15: 333-343$.

Eisenberg PR. Mechanisms of reocclusion after coronary thrombolysis. Z Kardiol 1993;82(Suppl. 2):175-178.

Garbini JL, Forster FK, Jorgensen JE. Measurement of fluid turbulence based on pulsed ultrasound techniques. Part 1. Analysis. I Fluid Mech 1982;118:445-470.

Goss SA. Johnston RL, Dunn F. Comprehensive compilation of 
cmpirical uitrasonic properties of mammalian tissues. $J$ Acous Soc Am 1978:64:423 -457.

Greenleat JF. Duck FA, Samayoa WF, Johnson SA. Ultrasonic data acquisition and processing system for atherosclerotic tissue characterization. IEEE Ultrason Symp Proceed 1974:738-743.

Iones SA, Fronek A. Effects of vibration on steady flow downstream of a stenosis. J Biomech 1988;21:903-914.

Kikuchi Y, Okuyama D, Kasai C. Yoshida Y. Measurement on the sound velocily and absorption of human blood in $1-10 \mathrm{MHz}$ Prequency range. Rec Elect Commun Eng Convers Tohoku Univ. 1972;41:152-159.

Ku DN. Giddens DP, Zarins CK, Glagov S. Pulsatile flow and atherosclerosis in the human carotid bifurcation: Positive correlation between plaque localization and low and oscillating shear stress. Arteriosclernsis 1985:5:293-302.
Latornell DJ, Pollard A. Some observations on the evolution of shear layer instabilities in laminar flow through axisymmetric sudden expansions. Phys Fluids 1986;29:2828 -2835.

Michael DH. Meniscus stability. Ann Rev Flud Mech 1981:13;184 215.

Newhouse VL. Furgason ES, Johnson GF. Wolf DA. The dependence of ultrasound Doppler bandwidth on beam geometry. IEEE Trans Sonics Ultrason 1980;27:50-59.

Stewart JT. Technology in interventional cardiology: Percutaneous transluminal coronary angioplasty. J Med Eng Technol 1991: $15: 99-106$

Thompson RS, Aldis GK, Linnett IW. Doppler ultrasound spectral power density distribution: Measurement artefacts in steady flow. Med Biol Eng Comput 1990; 28:60-66.

White FM. Viscous fluid flow. New York: McGraw-Hill, Inc. 1974. 